Ultrabroadband Airy light bullets

This content has been downloaded from IOPscience. Please scroll down to see the full text. 2014 J. Phys.: Conf. Ser. 497012003

(http://iopscience.iop.org/1742-6596/497/1/012003)

View the table of contents for this issue, or go to the journal homepage for more

Download details:

IP Address: 131.188.201.35

This content was downloaded on 17/08/2015 at 09:51

Please note that terms and conditions apply. 


\title{
Ultrabroadband Airy light bullets
}

\author{
Peeter Piksarv ${ }^{1}$, Andreas Valdmann ${ }^{1}$, Heli Valtna-Lukner ${ }^{1,2}$ \\ and Peeter Saari ${ }^{1,3}$ \\ ${ }^{1}$ Institute of Physics, University of Tartu, Riia 142, Tartu, 51014 Estonia \\ ${ }^{2}$ Max Planck Institute for the Science of Light, Günther-Scharowsky Str. 1, 91058 \\ Erlangen, Germany \\ ${ }^{3}$ Estonian Academy of Sciences, Kohtu 6, Tallinn, 10130 Estonia \\ E-mail: peeter.piksarv@ut.ee
}

\begin{abstract}
We present the measurements of the spatiotemporal impulse responses of two optical systems for launching ultrashort Airy pulses, incl. ultrabroadband nonspreading Airy beams whose main lobe size remains invariantly small over propagation. First, a spatial light modulator and, second, a custom refractive element with continuous surface profile were used to impose the required cubic phase on the input field. White-light spectral interferometry setup based on the SEA TADPOLE technique was applied for full spatio-temporal characterization of the impulse response with ultrahigh temporal resolution approaching a single cycle of the light wave. The results were compared to the theoretical model.
\end{abstract}

Keywords: Airy pulses, ultrafast measurement, non-diffractive waves

PACS numbers: 42.25.Bs, 42.65.Re, 42.79.-e 


\section{Introduction}

The family of non-diffracting or localized waves [1] has grown recently thanks to discovery of several wave-fields whose intensity maximum not only is transversely confined, but also moves along a curved trajectory during propagation in free space. The first and most well-known of such wavefields - the Airy beam-has attained enduring attention since its introduction in optics by Siviloglou and Christodoulides [2, 3]. Pulsed versions of the Airy beam have also been discussed and experimentally realized [47]. Although direct light-field modulation can be used to create Airy beams [8], a more common experimental setup involves imposing a cubic phase profile on the input Gaussian beam and performing an optical Fourier transform with a lens. The desired spatial phase modulation can be acquired, e.g., with spatial light modulators (SLM) [2], lithographically etched micro-optical phase plates [9], combinations of cylindrical lenses [10] and even by a purposeful use of lens aberrations [6]. Airy beams have been put to use for generation of curved plasma channels [11] and optical micromachining [12].

For many possible applications, it is important, that the main lobe of the beam remains laterally confined. In the monochromatic case, even the truncated version of Airy beam resists diffraction over propagation distances much larger than the Rayleigh length and its main intensity lobe retains its lateral confinement [2]. However, in the case of ultrashort pulses with broadband spectrum spreading over tens or hundreds of nanometres or spanning even over more than an octave, the dispersion becomes unavoidable. Therefore, the wavelength dependence of the system for creating ultrashort Airy pulses must be taken into consideration to understand the spatial and temporal properties of the main intensity lobe.

In this paper we study the impulse responses of two optical systems for launching ultrashort Airy pulses. Ultrashort Airy pulses shaped by an SLM and an optical element that has a continuous surface profile following a cubic function are compared. A spatialspectral interferometry technique based on the SEA TADPOLE method [13] was used to fully characterize the created Airy wave field with high spatial and temporal resolution.

\section{Theoretical models}

In general, a broadband 1-dimensional Airy beam can be expressed in the paraxial approximation as a superposition of its monochromatic constituents of wavenumber $k$ over spectrum $S(k)[4,5,14]$

$$
\Psi(x, z, t)=\int_{0}^{\infty} \mathrm{d} k S(k) \Phi(x, z, k) \mathrm{e}^{\mathrm{i} k(z-c t)},
$$

where

$$
\Phi(x, z, k)=\operatorname{Ai}\left[\frac{x}{x_{0}}-\left(\frac{z}{2 z_{0}}\right)^{2}+\mathrm{i} a \frac{z}{z_{0}}\right]
$$




$$
\begin{array}{r}
\times \exp \left[a \frac{x}{x_{0}}-\frac{a}{2} \frac{z^{2}}{z_{0}^{2}}+\mathrm{i} \varphi(x, z, k)\right], \\
\varphi(x, z, k)=\frac{x}{2 x_{0}} \frac{z}{z_{0}}-\frac{1}{12}\left(\frac{z}{z_{0}}\right)^{3}+\frac{a^{2}}{2} \frac{z}{z_{0}} .
\end{array}
$$

Here, $\operatorname{Ai}(\ldots)$ is the Airy function and $a$ is small parameter describing the lateral exponential decay of the infinite Airy trail. Scale of the beam is determined by lateral and longitudinal characteristic lengths $x_{0}$ and $z_{0}$. The parabolic wave-equation requires that $z_{0}=k x_{0}^{2}$. Additionally, the parameter $b_{0}=x_{0} / 4 z_{0}^{2}$ characterizes the parabolic trajectory of the main lobe of the Airy beam. The wave function of the 2D Airy beams is obtained as the product of two $1 \mathrm{D}$ wave functions $\Psi(x, y, z, t)=\Psi_{x}(x, z, t) \Psi_{y}(y, z, t)$.

The Airy beam is typically created with a lens performing an optical Fourier transform of the initial field [3]

$$
\Phi_{0}(\xi, k)=\mathrm{e}^{-a c_{0}^{2} \xi^{2}} \mathrm{e}^{\mathrm{i} \frac{1}{3}\left(c_{0}^{3} \xi^{3}-3 a^{2} c_{0} \xi-\mathrm{i} a^{3}\right)},
$$

were $\xi$ is the coordinate in the input plane of the lens. The lateral scale of the cubic phase function in the input plane is described by parameter $c_{0}$. The focal length $f$ of the lens imposes a relation $c_{0}=x_{0} k / f$ on the input and output fields. Four types of Airy pulses have been defined according to the dependence of $c_{0}$ on the wave-number $[4,5,7]$. Wavelength constituents of an Airy pulse can spatially overlap only in certain regions, which is determined by the four parameters of the Airy beam: $x_{0}, z_{0}, c_{0}$ and $b_{0}$. Type I Airy pulses have $x_{0}(k)=$ const, for type II $c_{0}(k)=$ const, type III pulses have $z_{0}(k)=$ const and for type IV $b_{0}(k)=$ const. The type of an Airy pulse is determined by properties of the optical system for creating it. It was previously shown that an SLM with a cubic phase mask containing discontinuities will produce type II Airy pulses that exhibit strong lateral dispersion [14].

For applications that require a spatially and temporally localized ultrashort pulse, type IV Airy beams would be most suitable as all its spectral components propagate along the same trajectory and their main intensity lobe is not subjected to lateral dispersion. The cubic phase term in (4) is proportional to $c_{0}^{3}$ and therefore for the nondispersing type IV Airy pulses with $c_{0} \propto k^{1 / 3}$, the phase modulation amplitude for different wavelengths in the Fourier plane is proportional to $k$. This could be achieved by using a suitably curved mirror as the cubic phase element. A refractive phase plate with a continuous cubic surface profile can also be used, but dispersion in the substrate material must be taken into account.

\section{Methods and experimental setup}

The underlying principle of our technique for measuring spatiotemporally resolved impulse responses is the well known spectrally resolved white-light interferometry. However, since the output field of an optical element generally depends not only on time and/or the longitudinal coordinate, but also on the transverse coordinates, the interferometry has to be generalized into three dimensions as follows. 
Let $E_{0}(t)$ be the electric field of the homogeneous, transversely coherent light beam in the input plane $z=0$ of the optical system. The field $E(\mathbf{r}, t)$ in the output halfspace $z>0$, which generally has acquired a 3D dependence on the field point coordinates $\mathbf{r}=(x, y, z)$ and a specific temporal dependence in every point, is given as temporal convolution

$$
E(\mathbf{r}, t)=\int_{0}^{\infty} h\left(\mathbf{r}, t^{\prime}\right) E_{0}\left(t-t^{\prime}\right) \mathrm{d} t^{\prime},
$$

where $h(\mathbf{r}, \tau)$ is the impulse response of the optical system for a location $\mathbf{r}$ behind it.

Let us suppose that somehow we have measured the cross-correlation function $K(\mathbf{r}, \tau)$ between the input and output fields $K(\mathbf{r}, \tau) \equiv\left\langle E(\mathbf{r}, t) E_{0}(t-\tau)\right\rangle$. Thereby we have also found out the impulse response function sought, since

$$
\begin{aligned}
K(\mathbf{r}, \tau) & =\left\langle\int_{0}^{\infty} h\left(\mathbf{r}, t^{\prime}\right) E_{0}\left(t-t^{\prime}\right) \mathrm{d} t^{\prime} E_{0}(t-\tau)\right\rangle= \\
& =\int_{0}^{\infty} h\left(\mathbf{r}, t^{\prime}\right)\left\langle E_{0}\left(t-t^{\prime}\right) E_{0}(t-\tau)\right\rangle \mathrm{d} t^{\prime}= \\
& =\text { const } \times h(\mathbf{r}, \tau),
\end{aligned}
$$

as far as the temporal dependence of the input field can be considered as a deltacorrelated stationary noise in which case $\left\langle E_{0}\left(t-t^{\prime}\right) E_{0}(t-\tau)\right\rangle \propto \delta\left(t^{\prime}-\tau\right)$ and the integration over $t^{\prime}$ drops out. In a general case, $K(\mathbf{r}, \tau)$ is the convolution of $h(\mathbf{r}, \tau)$ with the autocorrelation function of the input laser field and the impulse response can be recovered by a deconvolution method. As a matter of fact, the proportionality relation $h(\mathbf{r}, t) \propto K(\mathbf{r}, t)$ practically holds always whenever the spectrum of the input field is sufficiently "white", i.e., wider than the spectrum of $h(\mathbf{r}, t)$-in other words, wider than the support of the transfer function. Our setup follows the scheme of SEA TADPOLE, where the cross-correlation $K(\mathbf{r}, \tau)$ has been obtained through three procedures: (i) quadratic detection of the sum $E(\mathbf{r}, t)+E_{0}(t-\tau)$, where the $\tau$-dependence appears in the overlap region of the two beams tilted with respect to each other; (ii) temporal averaging due to the "slowness" of the detector (a CCD camera); (iii) spectral-domain separation of the cross-correlation term from the two non-oscillating autocorrelation terms. The spatial dependence of $h(\mathbf{r}, t) \equiv h(\mathbf{r}, \tau)$ is obtained by simply scanning the light-collecting tip of the signal-carrying fibre in the output half-space.

It should be pointed out that (6) in the case of time averaging is actually insensitive to the nature of the temporal dependence of the input light-be it deterministic ultrashort pulses or stationary noise or periodical bursts of temporally incoherent noise (which is the case of our set-up with a supercontinuum fibre laser). This favourable independence from the spectral phase of the light source follows from the basic principle of the spectrally resolved white-light interferometry, or-more generally-from the properties of time-averaged responses of linear systems.

The experimental setup for generating and measuring ultrabroadband Airy pulses is shown in figure 1. The measurement setup follows the SEA TADPOLE arrangement for spatiotemporal ultrashort pulse characterization using spatial spectral 


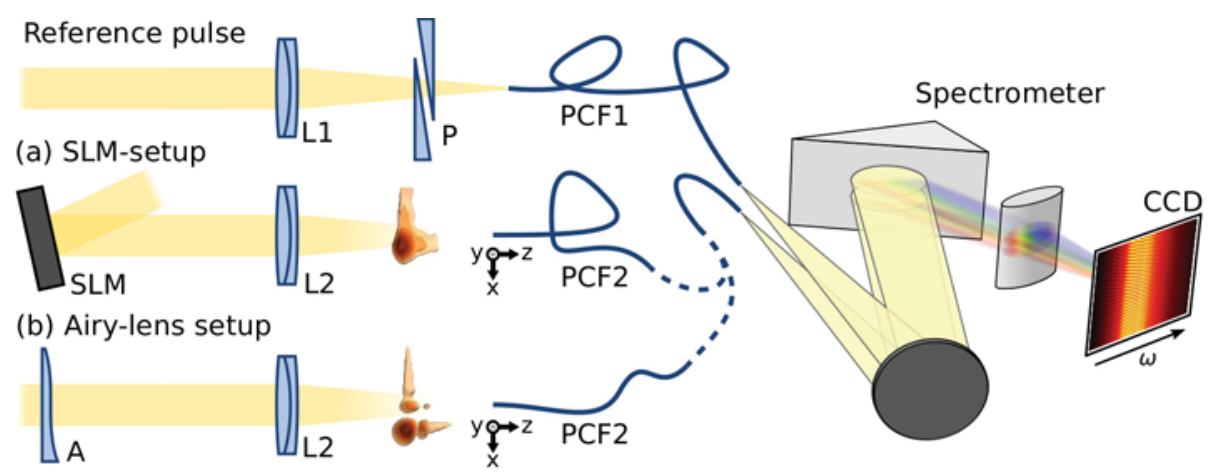

Figure 1. Experimental setup. Airy pulses were generated and measured in two setups: (a) using an SLM with wrapped cubic phase mask, (b) using a custom-made continuous phase element. An endlessly single mode photonic crystal fibre PCF2 samples a small region of the generated Airy fields. The reference is coupled into an identical fibre PCF1. The coupling lens L1 and the Fourier transforming lens L2 are of the same type. A wedge prism pair $\mathrm{P}$ was used to fine tune the dispersion between two arms of the inferferometer. The fibre outputs are placed on the entrance slit of the spectrometer at a small displacement resulting them to cross. A twodimensional spectral interferogram is recorded, and $E(\omega)$ is retrieved [13]. The fibre inputs on the measurement arm are scanned in three dimensions in order to measure the spatiotemporal field.

interferometry [13]. A more detailed description of the SEA TADPOLE method using supercontinuum laser source (Fianium SC400-2-PP) can be found in [15] and references therein. In short, the device involves sampling a small spatial region of the field under study with a single-mode photonic crystal fibre and recording its interference pattern with a reference pulse in a spectrometer from which the complex spectral response and hence also the impulse response is reconstructed at every point in space. The fibre is scanned both transversely along $x$ and $y$ axis and longitudinally along $z$. For longitudinal scanning, the delay on the reference arm was adjusted correspondingly to keep the relative path lengths of the interferometer arms constant. Our measurable spectral range was from $428 \ldots 1088 \mathrm{~nm}$ yielding a near-cycle temporal resolution of $2.5 \mathrm{fs}$. The spatial resolution is determined by the mode size of the fibres and is approximately $3 \mu \mathrm{m}$.

Type II Airy beam profile was created by a reflective SLM (Holoeye LC-R 2500) with an imposed cubic phase mask in both transverse orthogonal directions [14]. The SLM was placed in the focal plane of an achromatic lens with focal length of $f=500 \mathrm{~mm}$. A small uncompensated spectral phase term introduced by the thickness of the SLM and by the small inequality of the fibre lengths was determined with a zero phase mask on the SLM and subtracted from the measured spectral phase.

For generating type IV Airy pulses we chose to use a custom-made refractive phase element which can be directly placed into the beam path. The phase plate was fabricated using the PowerPhotonic LightForge service. The bulk dispersion of the fused silica substrate was compensated in the setup. Some material dispersion still remained as the 
phase element thickness varies, but this small perturbation does not cause any significant deviation of the created pulse from a pure type IV Airy pulse as it will be confirmed below.

In order to improve the transverse beam quality the laser beam was spatially filtered trough a single mode photonic crystal fibre and expanded using off-axis parabolic mirrors for type IV Airy pulse measurements. In addition, a pair of fused silica wedge prisms were added into the reference arm to precisely control the dispersion in the reference arm and compensate for the residual dispersion arising from the possible inequalities in the length of fibre and from the substrate of the phase element. Therefore any additional calibration of the device, i.e., subtracting a spectral phase of a blank substrate from the measurement results, was not necessary.

Two sets of achromatic lenses $(f=500 \mathrm{~mm}$ and $f=300 \mathrm{~mm})$ were used in the setup, effectively allowing to obtain desired parameters of the generated Airy pulses even with the given single phase element. As the linear dimensions of the generated Airy beam scale inversely proportionally to the focal length of the Fourier transforming lens, it is a convenient way to generate beam with a desired size of the focal spot.

The simulations were carried out by evaluating (1) using the measured spectrum. The parameters $x_{0}$ and $y_{0}$ were calculated from the phase mask displayed on the SLM and from the phase profile of the cubic phase element by taking into account the dispersion relation of the fused silica substrate of the element. It was assumed that the Fourier transform was performed by an ideal achromatic thin lens. The parameter $a$ for each wavelength was determined by measuring the beam profile with a separate CCD camera and a set of interference filters. These data were thereafter inter- and extrapolated to cover the full spectral range of the SEA TADPOLE.

\section{Results and discussion}

The measured spatiotemporal response of the systems with a lens of $f=500 \mathrm{~mm}$ for generating type II Airy pulses and type IV Airy pulses are shown and compared with simulations in figures 2 and 3, respectively. By comparing those figures it is clearly seen that the type II Airy pulse generated using an SLM exhibits strong lateral dispersion of the Airy maximum as the shorter wavelength constituents of the pulse are deflected more from the linear path. In contrast, the comparable size Airy maximum of the type IV pulse retains its spot size over the same propagation distance. With a lens of $f=500 \mathrm{~mm}$, the pulse cross section retained a distinct pattern typical to Airy beams throughout the longitudinal measurement range of $200 \mathrm{~mm}$ for both types of Airy pulses. However, near the Fourier plane of the lens at the distance $z=0 \mathrm{~mm}$ the Airy profile of the type II pulse was obstructed by the so-called zero-order of the SLM.

The propagation characteristics depend also on the focal length of the Fourier lens. The results of type IV Airy pulses generated with the continuous cubic phase mask and $f=300 \mathrm{~mm}$ lens are shown in figure 4 . With $f=300 \mathrm{~mm}$, the pulse exhibited stronger lateral acceleration, but the pattern on the cross section began to deteriorate 

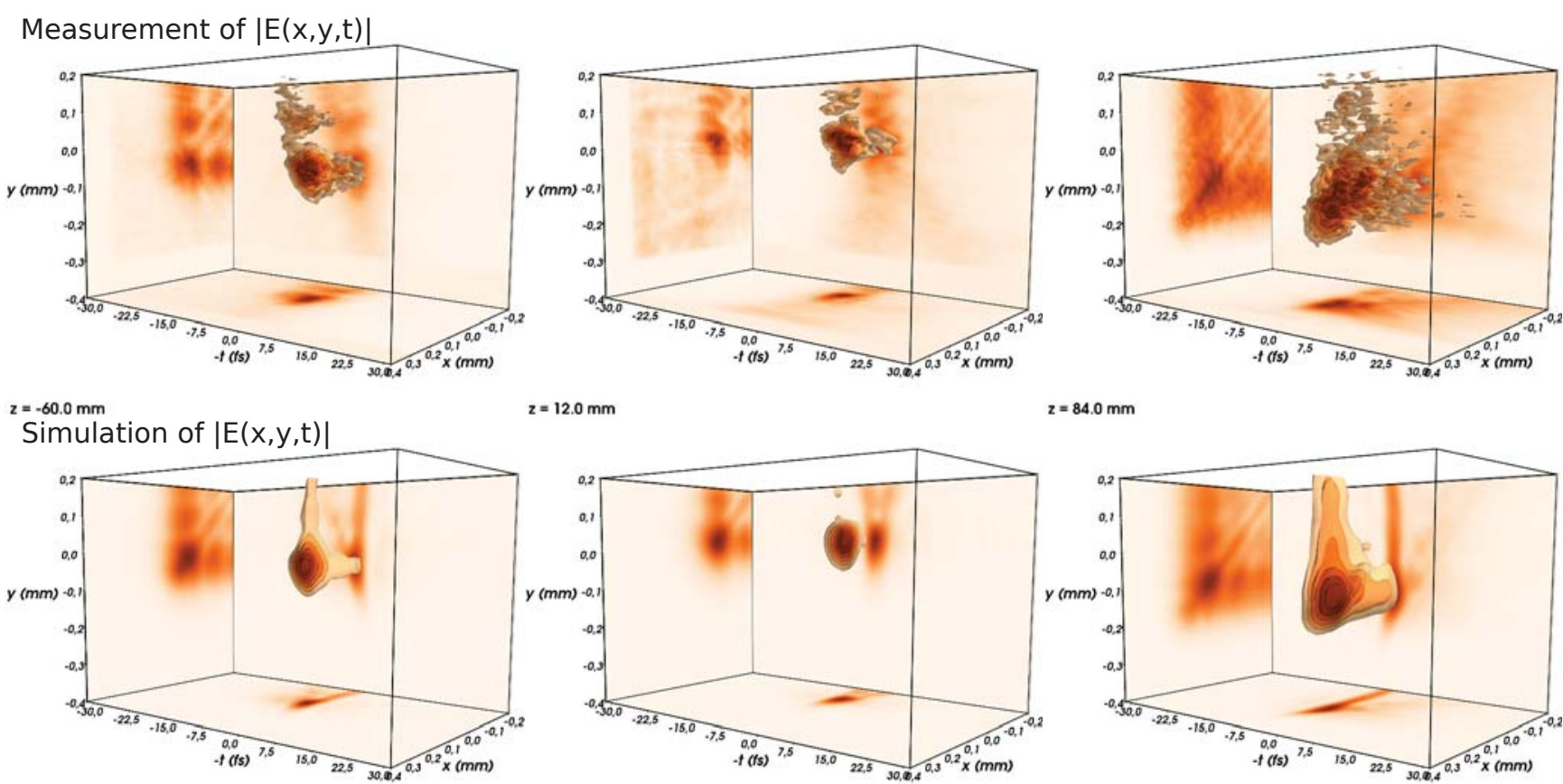

$z=12.0 \mathrm{~mm}$

$z=84.0 \mathrm{~mm}$
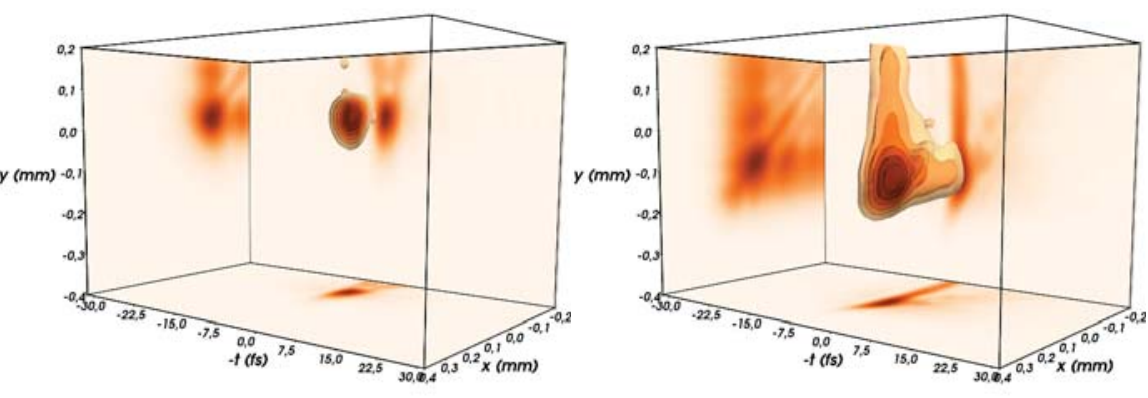

$z=-60.0 \mathrm{~mm}$

$z=12.0 \mathrm{~mm}$

$z=84.0 \mathrm{~mm}$

Figure 2. Measured (top) and simulated (bottom) impulse response of type II Airy pulse generator consisting of an SLM with wrapped cubic phase and a lens $f=500 \mathrm{~mm}$ at three distances. Colour represents the relative amplitude of the electric field isosurfaces. The images show projections of the pulse to the corresponding planes.
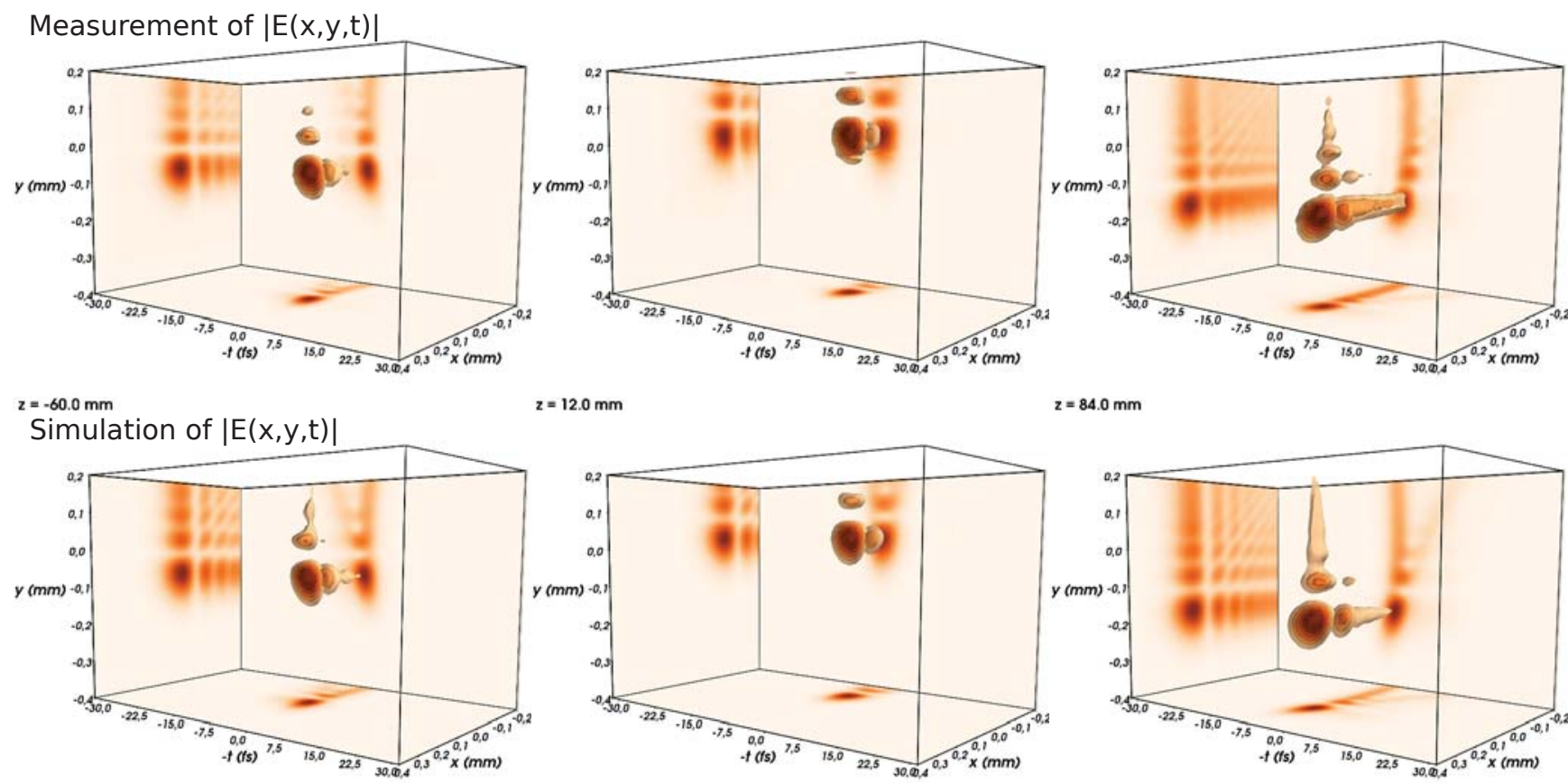

$z=12.0 \mathrm{~mm}$

$z=84.0 \mathrm{~mm}$
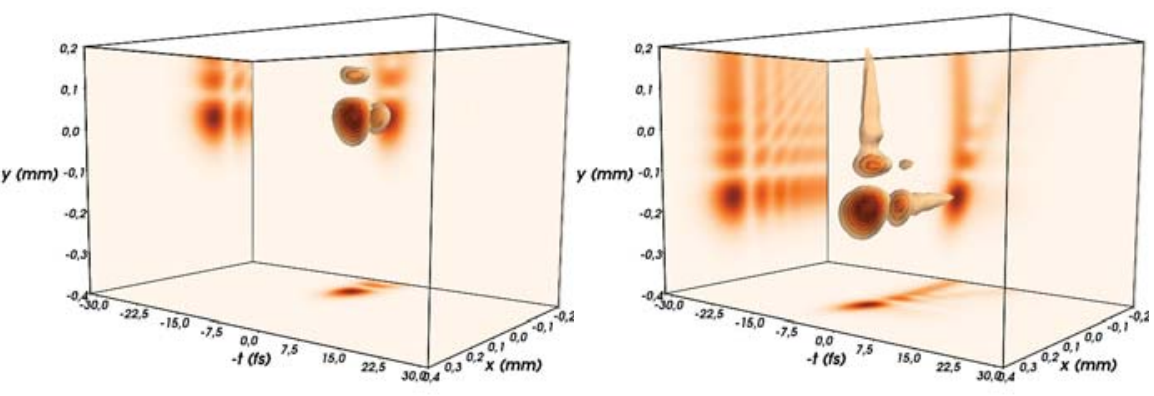

Figure 3. Measured (top) and simulated (bottom) impulse response of type IV Airy pulse generator consisting of an continuous cubic phase element and a lens $f=500 \mathrm{~mm}$ at three distances. 

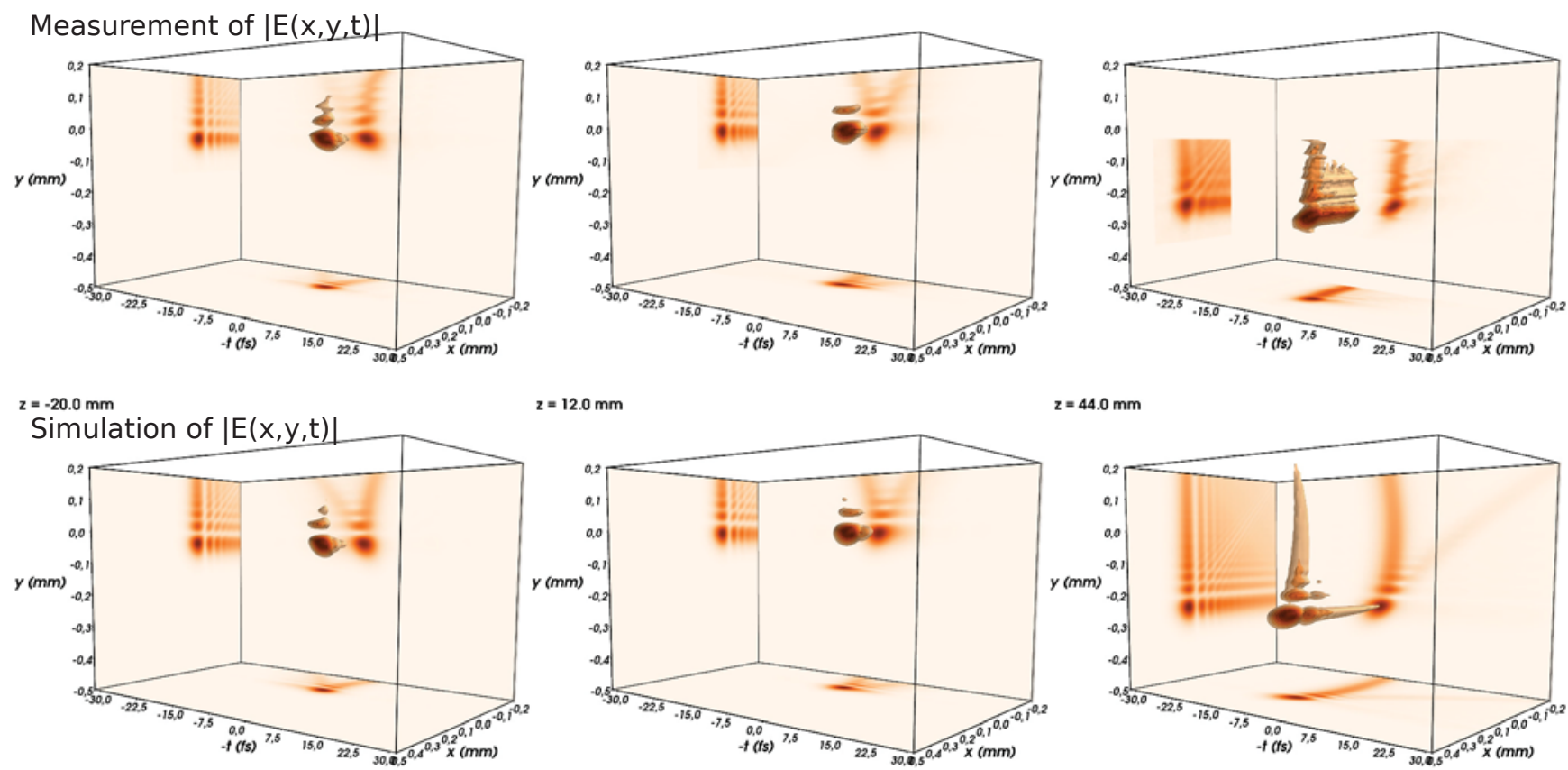

$z=12.0 \mathrm{~mm}$

$z=44.0 \mathrm{~mm}$
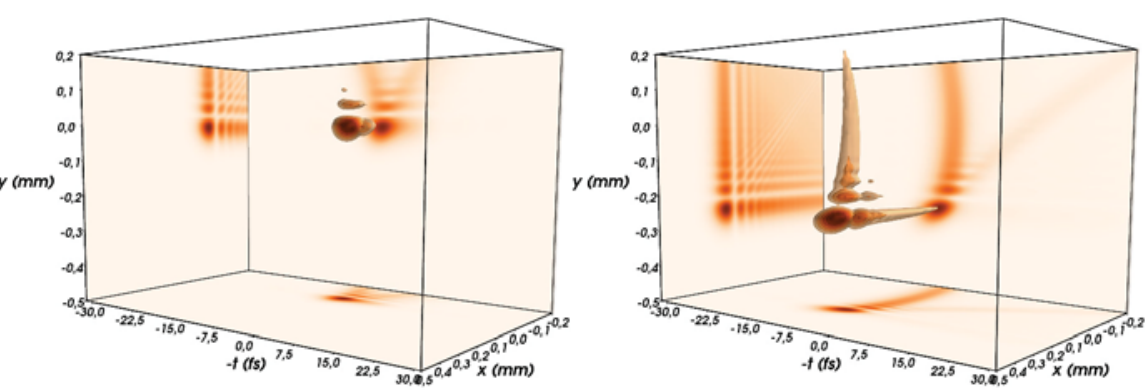

$z=-20.0 \mathrm{~mm}$

$z=12.0 \mathrm{~mm}$

$z=44.0 \mathrm{~mm}$

Figure 4. Measured (top) and simulated (bottom) impulse response of type IV Airy pulse generator consisting of the continuous cubic phase element and a lens $f=300 \mathrm{~mm}$ at three distances. The measurements were carried out only in the range $-225 \ldots 125 \mu \mathrm{m}$ respect to the intensity maximum with a $5 \mu \mathrm{m}$ step in both lateral directions which is two times smaller than in the previous measurements presented in this paper.

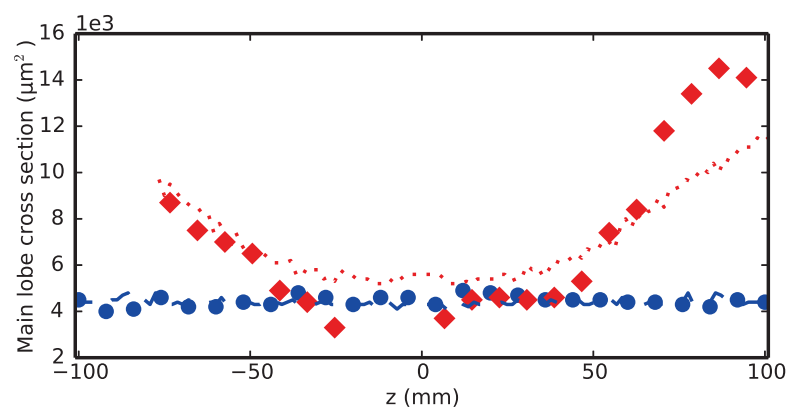

Figure 5. Measured (markers) and simulated (lines) main lobe cross section area of the ultrashort Airy pulse. Rhombuses and dotted line: Airy pulse generated by an SLM and Fourier lens with $f=500 \mathrm{~mm}$; circles and dashed line: nondispersing Airy pulse generated by special phase element and Fourier lens with $f=500 \mathrm{~mm}$.

when moving farther away from the apex of the parabolic trajectory along the $z$-axis. The main lobe was distinguishable throughout a propagation range of $100 \mathrm{~mm}$.

Spatial and temporal localization of the main intensity maximum during propagation was examined to demonstrate the nondispersive characteristics of the created pulse. The pulse intensity profile was averaged over time for each longitudinal measurement point and the transverse size of the main lobe was calculated as an area 
surrounded by the half maximum intensity contour. The measured and simulated cross section areas are shown in figure 5. For comparison, the main lobe cross section area of an Airy wave field created with an SLM was calculated. The main lobe size of the newly created pulse remains constant throughout the whole propagation range. This was not the case for type II pulses shaped with an SLM, where the main lobe quickly spreads out while moving away from the focus of the Fourier lens. This was caused by strong lateral dispersion as the wavelength constituents of the pulse showed a varying parabolic deflection rate during propagation.

The temporal duration of the pulse intensity maximum over the measured propagation range remained almost constant at $3.6 \mathrm{fs}$ and $3.3 \mathrm{fs}$ (FWHM) for type II and type IV Airy pulses, respectively. This, along with the aforementioned lateral confinement of the main lobe, suggests that creation of curvilinearly propagating localized ultrashort pulses, i.e., "light bullets", is feasible with the refractive phase element having a continuous cubic surface profile. When using a femtosecond laser to create the pulses, bulk dispersion of the cubic phase element can be compensated by adjusting the pulse compressor, which is typically present in the setup. It should be pointed out that the damage threshold of the used fused silica phase plate is much higher than of an SLM, which is promising for applications where high intensity pulses are needed.

\section{Conclusion}

In conclusion, we have measured the spatiotemporal impulse responses of optical systems for generating ultrabroadband Airy pulses. It was shown that an SLM with an wrapped cubic phase mask creates type II Airy pulses, which have identical spectral phase distribution over the beam cross section after the SLM. Furthermore, it was shown that a continuous cubic phase plate creates type IV Airy pulses, which have laterally nondispersing main lobe that does not spread out over its propagation range. The temporal duration and cross section area of the main lobe of the pulse remained constant during propagation. Properties of the created pulse can be adjusted by selecting a suitable Fourier lens. Choosing a lens with a shorter focal length will result in Airy pulses with stronger later acceleration and smaller main lobe, but aberrations are more noticeable at shorter propagation distances. The presented results are important for real generation of near-single-cycle pulses that follow a curved trajectory, which can be used, e.g., for exploring nonlinear optical processes, high-precision micromachining, fluorescence microscopy, and particle manipulation. Finally, this work emphasises the important differences between the responses of diffractive and refractive optical elements under broadband illumination. 


\section{Acknowledgments}

This work has been supported by the Estonian Science Foundation grant 7870 and by the Estonian Centre of Excellence in Research "Centre for Nonlinear Studies" receiving funding from the European Regional Development Fund under project 3.2.0101.110037. A. V. has been partially supported by the graduate school "Functional Materials and Technologies" receiving funding from the European Social Fund under project 1.2.0401.09-0079.

\section{References}

[1] H. E. Hernández-Figueroa, E. Recami, and M. Zamboni-Rached, editors. NonDiffracting Waves. Wiley-VCH, 2013.

[2] G. A. Siviloglou and D. N. Christodoulides. Accelerating finite energy Airy beams. Opt. Lett., 32(8):979-981, 2007.

[3] G. A. Siviloglou, J. Broky, A. Dogariu, and D. N. Christodoulides. Observation of accelerating Airy beams. Phys. Rev. Lett., 99(21):213901, 2007.

[4] P. Saari. Laterally accelerating Airy pulses. Opt. Express, 16(14):10303-10308, 2008 .

[5] P. Saari. Airy pulse - a new member of family of localized waves. Laser Physics, 19(4):725-729, 2009.

[6] D. Abdollahpour, S. Suntsov, D. G. Papazoglou, and S. Tzortzakis. Spatiotemporal Airy light bullets in the linear and nonlinear regimes. Phys. Rev. Lett., 105(25):253901, 2010.

[7] Y. Kaganovsky and E. Heyman. Airy pulsed beams. J. Opt. Soc. Am. A, 28(6):1243-1255, 2011.

[8] D. M. Cottrell, J. A. Davis, and T. M. Hazard. Direct generation of accelerating airy beams using a 3/2 phase-only pattern. Opt. Lett., 34(17):2634-2636, 2009.

[9] J. Wang, J. Bu, M. Wang, Y. Yang, and X. Yuan. Generation of high quality Airy beams with blazed micro-optical cubic phase plates. Appl. Opt., 50(36):6627-6631, 2011.

[10] B. Yalizay, B. Soylu, and S. Akturk. Optical element for generation of accelerating Airy beams. J. Opt. Soc. Am. A, 27(10):2344-2346, 2010.

[11] P. Polynkin, M. Kolesik, J. V. Moloney, G. A. Siviloglou, and D. N. Christodoulides. Curved plasma channel generation using ultraintense Airy beams. Science, 324(5924):229-232, 2009.

[12] A. Mathis, F. Courvoisier, L. Froehly, L. Furfaro, M. Jacquot, P. A. Lacourt, and J. M. Dudley. Micromachining along a curve: Femtosecond laser micromachining of curved profiles in diamond and silicon using accelerating beams. Appl. Phys. Lett., 101(7):071110, 2012. 
[13] P. Bowlan, P. Gabolde, A. Shreenath, K. McGresham, R. Trebino, and S. Akturk. Crossed-beam spectral interferometry: a simple, high-spectral-resolution method for completely characterizing complex ultrashort pulses in real time. Opt. Express, 14(24):11892-11900, 2006.

[14] P. Piksarv, A. Valdmann, H. Valtna-Lukner, R. Matt, and P. Saari. Spatiotemporal characterization of ultrabroadband Airy pulses. Opt. Lett., 38(7):1143-1145, 2013.

[15] P. Piksarv, H. Valtna-Lukner, A. Valdmann, M. Lõhmus, R. Matt, and P. Saari. Temporal focusing of ultrashort pulsed Bessel beams into Airy-Bessel light bullets. Opt. Express, 20(15):17220-17229, 2012. 